\title{
Body Mass Index Predicts Fighting Ability in Female UFC Fighters, but Facial Width-to-Height Ratio May Not
}

\author{
Jaime L. Palmer-Hague ${ }^{1}$ (D) Samuele Zilioli ${ }^{2}$. \\ Justin Jagore $^{3}$ - Tara L. DeLecce ${ }^{2}$
}

Received: 13 July 2015 / Revised: 24 August 2015 / Accepted: 26 August 2015 /

Published online: 9 September 2015

(C) Springer International Publishing 2015

\begin{abstract}
Several lines of evidence suggest that facial-width-to-height ratio (fWHR) provides an accurate cue to men's formidability, thus providing observers with a reliable estimate of their potential success in an interpersonal physical conflict. The degree to which fWHR provides the same information in women's faces, however, remains unclear. In fact, morphological characteristics, such as body size, may be more effective indicators of formidability in women. In the present study, we analyzed the effects of both fWHR and body mass index (BMI) on total number of fights and total number of wins in female competitive Ultimate Fighting Challenge (UFC) fighters. After controlling for number of active years fighting, we found that BMI significantly predicted number of wins in female fighters. The effects of fWHR were not significant, but warrant replication with a larger sample. These results suggest that in women, body size, rather than fWHR, may be a more accurate indicator of success in aggressive interpersonal conflicts. Our findings contribute to a growing body of literature that shows distinct differences in men and women's interpersonal aggressive and dominance behavior, as well as their morphological indicators.
\end{abstract}

Keywords Facial width to height ratio (fWHR) - Formidability · Physical strength · Female faces $\cdot$ Body mass index (BMI)

Jaime L. Palmer-Hague

jaime.palmerhague@twu.ca

1 Department of Psychology, Trinity Western University, 7600 Glover Road, Langley, BC V2Y 1Y1, Canada

2 Department of Psychology, Wayne State University, 5057 Woodward Avenue, 7th Floor, Detroit, MI 48202, USA

3 Department of Psychology, Simon Fraser University, 8888 University Drive, Burnaby, BC V5A 1S6, Canada 


\section{Introduction}

The ability to assess the potential threat of another individual is important for effective human social interaction. A growing body of literature suggests that the human face is a reliable indicator of various physiological, psychological, and behavioral characteristics in men and women, and recently, researchers have identified facial width-to-height ratio (fWHR), or the ratio of the bizygomatic width to height of the upper face (Weston et al. 2007), as a possible signal of formidability (e.g., Zilioli et al. 2014). Independent of body size, fWHR may provide an indication of an individual's fighting ability, thus providing others with a means to assess their own probability of success in a competitive encounter with them. Although fWHR has been consistently associated with aggressive behavior in men (reviewed in Haselhuhn et al. 2015), these relationships are either non-existent (Carré and McCormick 2008; Haselhuhn and Wong 2011; Stirrat and Perrett 2010) or small in women (Geniole et al. 2012). Indeed, women exhibit significantly lower levels of overt aggression compared to men (reviewed in Campbell and Cross 2012) and facial indicators of formidability may not have been selected for in this population. Other anthropometric features, such as body size, that provide direct indication of women's formidability might be more effective, but no studies have directly compared fWHR and body size as predictors of aggressive behavior in this population. In the present study, we examined whether fWHR, compared to body size, were more accurate indicators of fighting ability in female professional fighters.

Although fWHR has been linked to a variety of aggressive behaviors in men, similar relationships in women have not been consistently shown. Lefevre et al. (2014) found that self-reported verbal aggression in women was positively associated with their fWHR, but the same association has not been found for several other dominance behaviors. For example, in men, but not women, greater fWHR has been positively associated with deceptive behavior and cheating (Haselhuhn and Wong 2011), exploitation of others' trust (Stirrat and Perrett 2010), and overt aggression towards others (Carré and McCormick 2008; but see Deaner et al. 2012; Özener 2012). Men with greater fWHR may also be more successful in physically aggressive challenges (i.e., fights in Ultimate Fighting Championship (UFC) competitions) (Trebicky et al. 2015; Zilioli et al. 2014). In addition, men, but not women, with greater fWHR may be less likely to die in a physically violent encounter (Stirrat et al. 2012), suggesting that they may be more successful when faced with physical danger. Given that fWHR may be a sexually dimorphic (Weston et al. 2007; Carré and McCormick 2008; but see Özener 2012; Lefevre et al. 2012; Kramer 2015) and testosterone-linked trait (Lefevre et al. 2013), and that men tend to engage in more aggressive behavior than women do (reviewed in Archer 2006), these discrepancies may not be surprising. It is plausible that differences in fWHR amongst women are smaller in magnitude than they are for men, making them more difficult to detect.

An alternate position, however, may be that fWHR is actually not an indicator of formidability in women. Several studies have shown that certain aspects of women's behavior can be accurately assessed from their faces, but none have included an fWHR measurement. For example, after controlling for age, facial expression, and attractiveness and masculinity ratings, Rule and Ambady (2009) showed that female chief executive officers who were rated as having higher leadership quality were more likely to have led companies with higher annual profits, and those who were rated as 
more dominant earned higher salaries, but the facial features raters used to make these judgments was not assessed. Similarly, Quist et al. (2011) showed that women who scored higher in self-reported dominance had faces that were rated as more masculine, but they did not assess which facial markers were used to make these assessments. In addition, although Geniole et al. (2012) found that fWHR and ratings of aggressiveness in women's faces were moderately positively correlated, this relationship was significantly stronger for male faces, suggesting that observers may have simply generalized a male facial cue to female faces.

One possible indicator of formidability in women is body size. Several studies have demonstrated a positive relationship between body size and aggressive behavior. For example, Pinhey (2002) found that body mass index (BMI) was a significant predictor of whether adolescent women reported both participating in sports as well as being in at least one fight in the previous year. Similarly, Gallup and Wilson (2009) showed that BMI was positively associated with the tendency to demean, diminish, and embarrass others (but not acts of physical aggression) in a sample of adolescent women. This suggests that body size could be related to a variety of different forms of aggressive behavior

Results from other studies suggest that objective raters' perceptions of women's formidability are also associated with body size. Using a different sample of women, Gallup and Wilson (2009) found that objective raters perceived the faces of adolescents with higher BMI to be more aggressive. Similarly, Sell et al. (2009) found that objective raters could accurately predict women's handgrip strength from photographs of their faces, and were even more accurate when making judgments from photographs of full bodies. In addition, Fessler et al. (2014) showed that when naïve participants were asked to choose which target body best depicted a described risk-prone woman, they were significantly more likely to choose a taller and larger target than when asked to choose which woman depicted a described risk-averse woman. Body size, then, may be used as a cue to women's formidability, including strength, aggressive tendency, and willingness to engage in activities with high risk of physical harm.

Although the results of a recent meta-analaysis showed a positive association between fWHR and BMI in women (Geniole et al. 2015), a relative lack of data from studies investigating the relationship between these variables and women's threat and dominance behavior precludes the conclusion that either is an exclusive indicator of their formidability. In order to determine whether fWHR or body size provides a more accurate indication of formidability in women, we investigated whether fWHR and BMI were significant predictors of fighting ability in professional female fighters. We used information available from female fighters associated with the Ultimate Fighting Championship ${ }^{\circledR}$ (UFC), the world's largest organization for mixed-martial arts, a style of full-contact combative sport that blends striking and grappling techniques in an effort to physically overpower and subdue an opponent. In the UFC, very little regulation is placed upon the methods used by fighters in competition, and winners move to subsequent challenges with other fighters whereas recurring losers are quickly eliminated from further competition. The sample of female fighters used was therefore ideal in that it reflected a sort of "Darwinian" type of competitive environment, provided objective measurements of fighting ability, and assured appropriate variability within a subsample of women depicting physically aggressive behavior. 


\section{Method}

Facial width-to-height ratio, BMI, and fight statistics were analyzed from female fighters associated with UFC up to November 2014. Using an approach similar to recent studies involving male UFC fighters (Zilioli et al. 2014; Baker and Schorer 2013), we analyzed four measures of formidability: number of active years professional fighting, number of fights, number of wins, and proportion of wins (number of wins/number of fights). Such an approach takes into consideration bias inherent in this data set whereupon matches are determined in a non-random fashion (e.g., best fighters fight each other) and new fighters having a disproportionate number of wins (i.e., newly joined UFC fighters have won many fights in their previous, less challenging organizations).

Measures of formidability (i.e., number of active years professional fighting, number of wins, number of fights, and proportion of wins (number of wins/number of fights)) as well as heights and weights for 54 female fighters were collected from the official UFC website (www.ufc.com) Body mass index was calculated by entering each fighter's height and weight into an online BMI calculator hosted by the National Heart, Lung, and Blood Instistute (NHLBI). Facial photographs of each athlete were also taken from the UFC website. Close-up photographs were missing for four individuals and 10 pictures were excluded because the zygions were not visible (e.g., long hair covering them), thus measurements were available for 40 fighters. Facial width-toheight ratio for each fighter was measured as per Carré and McCormick (2008).

We used Generalized Linear Models with Maximum Likelihood Estimation and linear regressions (ordinary least square) to test whether BMI and fWHR predicted formidability (i.e., number of fights, number of wins, and proportion of wins). Number of fights and number of wins are count variables and were therefore modeled using Poisson regression with robust estimator covariance. On the other hand, proportion of wins was modeled using linear regression (for a similar approach, see Trebicky et al. 2013). To facilitate interpretation, continuous predictors were standardized. The threshold for statistical significance in all analyses was set at $p \leq .05$.

\section{Results}

Descriptive statistics are reported in Table 1.

Table 1 Descriptive statistics for female UFC fighters

\begin{tabular}{|c|c|c|c|c|}
\hline & $\mathrm{N}$ & Mean (or \%) & SEM & $\mathrm{SD}$ \\
\hline Weight class (Bantamweight) & 54 & $57.4 \%$ & - & - \\
\hline Age & 54 & 26.67 & .52 & 3.85 \\
\hline Body mass index (BMI) & 54 & 20.78 & .20 & 1.44 \\
\hline Facial width-to-height ratio (fWHR) & 40 & 1.88 & .02 & .13 \\
\hline Number of fights & 54 & 11.33 & .88 & 6.47 \\
\hline Number of wins & 54 & 8.24 & .61 & 4.51 \\
\hline Proportion of wins & 54 & .75 & .02 & .18 \\
\hline
\end{tabular}




\section{BMI, fWHR and Formidability}

Pearson bivariate correlations revealed that fWHR and BMI were positively correlated $(r=.571, p=.003)$, but there were no significant associations found between fWHR and number of fights $(r=.137, p=.400)$, number of wins $(r=.106, p=.514)$, or proportion of wins $(r=-.092, p=.571)$. In contrast, BMI was significantly positively correlated with number of fights $(r=.372, p=.006)$ and number of wins $(r=.456, p=.001)$, but not proportion of wins $(r=.188, p=.173)$. Number of active years fighting was positively correlated with BMI $(r=.278, p=.041)$ and negatively correlated with proportion of wins $(r=-.268, p=.050)$. This latter finding was expected; fighters are required to have won virtually all fights in their previous organization in order to join UFC, and therefore exhibit a disproportionate number of wins compared to fighters with longer UFC membership. In order to account for this bias, we included number of years fighting as a covariate in subsequent regression analyses. Results obtained from analyses without number of active years fighting as a covariate are also provided for comparison.

First, we performed Poisson regression analyses with fWHR, BMI, and number of active years fighting as predictors and either number of fights or number of wins as the dependent variable. These analyses revealed that BMI was positively associated with both number of fights $(b=.154, S E=.0541,95 \% \mathrm{CI}:(0.048,0.260), p=.004)$ and number of wins $(b=.231, S E=.0545,95 \% \mathrm{CI}$ : $(0.125,0.338), p<.001)$, while fWHR was not associated with number of fights $(b=0.018, S E=0.0573,95 \%$ CI: $[-0.094$, $0.131], p=.748)$ or number of wins $(b=-0.043, S E=0.0616,95 \%$ CI: $(-0.163,0.078)$, $p=.488)$. As expected, number of active years fighting was positively associated with both total number of fights $(b=.407, S E=.0406,95 \% \mathrm{CI}:(0.327,0.487), p<.001)$ and total number of wins $(b=.342, S E=.0433,95 \% \mathrm{CI}:(0.257,0.427), p<.001)$. Partial correlations for fWHR and BMI controlling for number of active years fighting are provided in Table 2. ${ }^{1}$ Poisson regression analyses with fWHR and BMI, but not number of active years fighting, as predictors also revealed a significant effect of BMI on both number of fights $(b=.236, S E=.0932,95 \% \mathrm{CI}:(0.053,0.419), p=.010)$ and number of wins $(b=.305, S E=.0846,95 \% \mathrm{CI}:(0.139,0.471), p<.001)$. There was no significant association between fWHR and number of fights $(b=-.023, S E=.0886,95 \% \mathrm{CI}$ : $(-0.196,0.151), p=.799)$ or number of wins $(b=-.072, S E=.0797,95 \% \mathrm{CI}$ : $(-0.229,0.084), p=.363)$.

Next, we performed linear regression analyses using fWHR, BMI, and number of active years fighting as predictors and proportion of wins as the dependent variable. The effect of BMI approached significance $(b=.059, S E=.031,95 \%$ CI: $(-0.004$, $0.122), p=.062)$, but there was no significant effect of fWHR $(b=-.046, S E=.031$, $95 \%$ CI: $(-0.109,0.017), p=.141)$. The overall model also failed to reach significance $(F(3,36)=2.439, p=0.080)$. Partial correlations controlling for number of active years fighting are shown in Table 2. Linear regression analyses with BMI and fWHR, but not number of active years fighting, as predictors also showed no association between BMI $(b=.049, S E=.032,95 \%$ CI: $(-0.016,0.114), p=.135)$ or fWHR $(b=-.039, S E=.032$, $95 \%$ CI: $(-0.104,0.258), p=.232)$ and proportion of wins. The overall model was also not significant $(F(2,37)=1.338, p=0.275)$. It is important to note, however, that these

\footnotetext{
${ }^{1}$ Partial correlations for BMI were run using the full sample of faces $(n=54)$.
} 
Table 2 Partial correlations between fWHR, BMI, and formidability while controlling for number of active years fighting

fWHR

BMI

fWHR

BMI

$.489^{* * *}$

Number of fights

$.301^{\dagger}$

$.262^{\dagger}$

Number of wins

.201

$.384^{* * *}$

Proportion of wins

$-.108$

$.284^{*}$

Note: ${ }^{*} p<.05 ;{ }^{* *} p<.01,{ }^{\dagger} p=.06$

results could be due to the reduction in sample size for fWHR as suggested by the fact that when similar analyses were conducted on the full sample of fighters, a significant association emerged between BMI and proportion of wins (see below for more details).

\section{BMI and Formidability}

In order to assess the influence of reduced sample size, we conducted the same analyses as above with the full sample $(n=54)$ (i.e., including those without fWHR measurements), we performed Poisson regression analyses with BMI and number of active years fighting as predictors, and either number of fights or number of wins as the dependent variable. The results of these analyses confirmed the effect of BMI on formidability reported above. BMI was associated with both total number of fights $(b=.097 S E=.0425,95 \%$ CI: $(0.014,0.181), p=.022)$ and total number of wins $(b=.150 S E=.041,95 \%$ CI: $(0.070,0.231), p<.001)$ (Fig. 1$)$. Number of active years was also associated with total number of fights $(b=.402 S E=.0323,95 \%$ CI: (0.339, $0.466), p<.001)$ and total number of wins $(b=.327 S E=.039,95 \% \mathrm{CI}:(0.251,0.404)$, $p<.001)$. Partial correlations controlling for number of active years fighting are shown in Table 2. Poisson regression with BMI, but not number of active years fighting, as a predictor also revealed a significant effect of BMI on both number of fights $(b=.202$ $S E=.0491,95 \% \mathrm{CI}:(0.106,0.298), p<.001)$ and number of wins $(b=.235 S E=.0456$, 95 \% CI: $(0.146,0.325), p<.001)$.

We then performed a linear regression with BMI and number of active years fighting as predictor variables and proportion of wins as the dependent variable. Both BMI $(b=.050 S E=.024,95 \%$ CI: $(0.002,0.098), p=.039)$ and number of active years fighting $(b=-.061 S E=.024,95 \% \mathrm{CI}:(-0.109,-0.013), p=.013)$ significantly predicted proportion of wins, suggesting that the restricted sample size did not provide sufficient power to detect these effects $(F(2,51)=4.396, p=0.017) .{ }^{2}$ Linear regression analyses with BMI, but not number of years fighting, as a predictor revealed that BMI was not a significant predictor of proportion of wins $(b=.030 S E=.022,95 \% \mathrm{CI}$ : $(0.339,0.466), p=.173)$, and the overall model was not significant $(F(1,52)=1.912, p=$ $0.173)$.

\footnotetext{
${ }^{2}$ In martial arts tournaments only individuals of the same weight class are permitted to fight each other. Using fighters weight class $(0=$ Strawweight; $1=$ Bantamweight $)$ as a covariate did not alter the significance of any reported findings.
} 


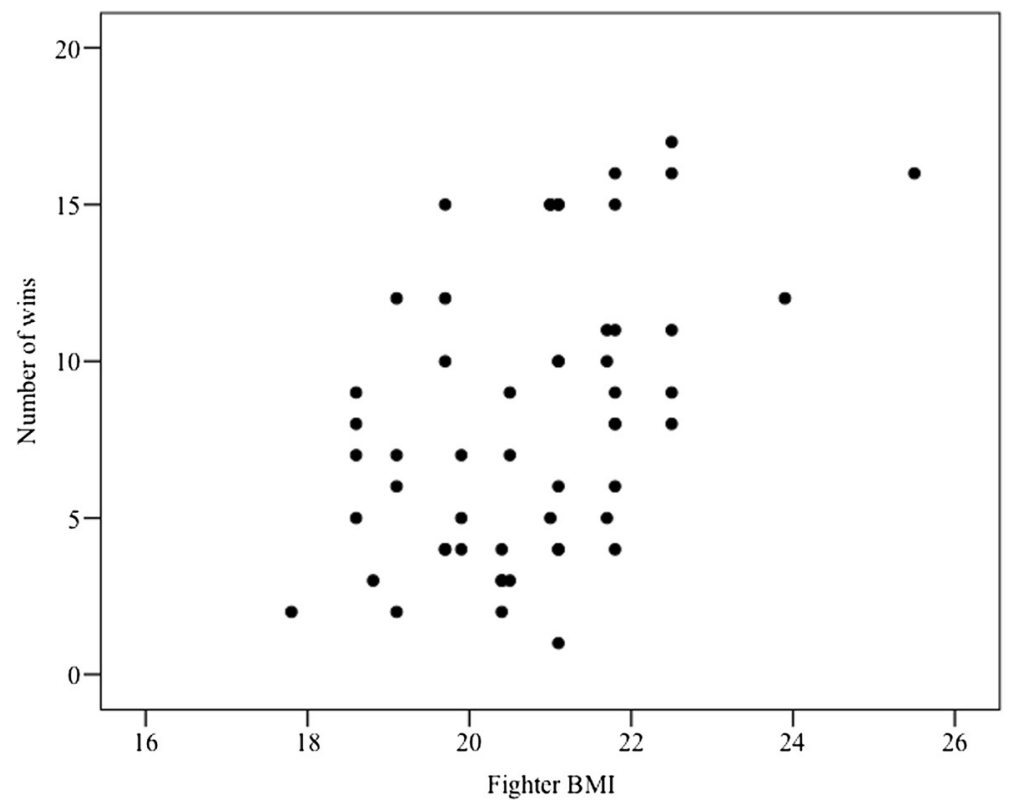

Fig. 1 Number of wins as a function of female fighters' body mass index (BMI)

\section{Discussion}

Our analysis of fighting ability in female UFC fighters revealed that body size may be a better indicator of fighting ability than fWHR in women. Specifically, after controlling for number of active professional years fighting, we found that women's BMI was a significant predictor of the number of fights, number of wins, and proportion of wins that they had achieved. All of these effects also remained significant when number of active years fighting was not included as a covariate except for proportion of wins, suggesting that BMI is exclusively associated with fighting ability. In contrast, fWHR was not a significant predictor of these outcomes, providing additional evidence that fWHR might not be the most salient cue of formidability in women.

The positive correlation observed between fWHR and BMI presents the possibility that fWHR provides information about fighting ability via its association with BMI; however, we did not find exclusive relationships between fWHR and measures of fighting ability, suggesting that it is not a useful cue in this regard. It is worthwhile to note, however, that some of our null findings could have been due to lack of power in our relatively small sample of facial photographs $(n=40)$. Similarly, because women UFC fighters represent a small subsection of the female population, it is likely that the range of fWHR measurements in our sample was narrow, reducing the likelihood of us detecting a significant association. Indeed, we did see the relationship between BMI and proportion of wins increase in significance from $p=.062$ in the smaller sample to $p=.017$ in the larger sample, suggesting that we may have seen a similar effect for fWHR $(p=.141)$. Verification of our results in larger samples therefore precludes a definitive conclusion. 
Nevertheless, our results are in line with previous work showing that body size is positively associated with physical strength in women (e.g., Miyatake et al. 2012), that it indicates physical strength (Sell et al. 2009) and health (Coetzee et al. 2011) to others, and that it correlates with both overt (Pinhey 2002) and indirect (Gallup and Wilson 2009) aggressive behavior. Given that higher testosterone is also associated with higher BMI in women (e.g., Sowers et al. 2001), there is likely a functional relationship between testosterone, social status, and body size in this population. However, whereas women's dominance and aggression have been positively associated with testosterone in some studies (e.g., Cashdan 2003; Grant and France 2001), others have found negative associations between androgens and social status (e.g., Cashdan 1995), suggesting that the contexts of this relationship may be different for women than it is for men. Indeed, it has been hypothesized that women are more likely to compete indirectly for mates (i.e., reducing the attractiveness of other females, gossiping) rather than engaging in physical altercations with them (Campbell and Cross 2012). Investigation into the endocrinology of, precursors to, and results of female aggressive and competitive behavior clearly warrants further study.

Our results are also in keeping with previous research that has not shown effects of fWHR on female aggressive and fighting behavior (Carré and McCormick 2008; Haselhuhn and Wong 2011; Stirrat and Perrett 2010; Stirrat et al. 2012; but see Geniole et al. 2012), thus providing additional evidence that fWHR might not be an effective indicator of their formidability. However, in addition to the possibility that we were simply unable to detect effects of fWHR due to sample size, the results of a recent meta-analysis showed that BMI and fWHR are positively significantly correlated (Geniole et al. 2015), suggesting that fWHR may still have an important association with formidability in women. Indeed, Lefevre et al. (2014) showed that women's selfreported verbal aggression was positively related to fWHR. Additional research with women's faces is clearly needed.

Higher adiposity could potentially mask researchers' ability to assess fWHR in women's faces compared to men's, but it is more likely that sex differences in facial architecture are due to differences in levels of circulating steroid hormones both developmentally and in adulthood (i.e., higher testosterone in males compared to females, higher estrogen in females compared to males). As Lefevre et al. (2013) have suggested, it is plausible that women's facial characteristics are entirely different than men's. Though it is important to note that neither assessed fWHR, at least two other studies have shown that women's faces reveal aspects of their dominance and aggressive behavior (Quist et al. 2011; Rule and Ambady 2009). This presents the possibility that additional facial markers might indicate women's formidability. Adiposity, for example, is estrogen dependent and has been shown to vary negatively with ratings of facial masculinity in women (Law Smith et al. 2006). Interestingly, participants associate lower facial adiposity with better leadership skills in women (Re and Perrett 2014), suggesting that it might also serve as a cue to women's dominance and aggressive behavior.

Although our results suggest that body size is a reliable indicator of female fighting ability, it is important to note that our sample was limited to a small number of professional UFC fighters, and may not be generalizable to all women. As women are less likely to engage in physical aggression than other types of competitive behavior (e.g., Cashdan 2003), the average woman may not engage in repeated physical fights, if 
ever at all. Thus, it will be important for future studies to replicate our findings both in groups of women who are not professional fighters, as well as with other types of female status behaviors.

In sum, we show here that in a sample of female UFC fighters, body size, but not fWHR, is a reliable indicator of fighting ability and may be an accurate cue to formidability in this population.

\section{References}

Archer, J. (2006). Testosterone and human aggression: an evaluation of the challenge hypothesis. Neuroscience and Biobehavioral Reviews, 30(3), 319-345.

Baker, J., \& Schorer, J. (2013). The southpaw advantage? Lateral preference in mixed martial arts. PLoS ONE, 8, e79793. doi:10.1371/journal.pone.0079793.

Carré, J. M., \& McCormick, C. M. (2008). In your face: facial metrics predict aggressive behaviour in the laboratory and in varsity and professional hockey players. Proceedings of the Royal Society of London B: Biological Sciences, 275(1651), 2651-2656.

Cashdan, E. (1995). Hormones, sex, and status in women. Hormones and Behavior, 29, 354-366.

Cashdan, E. (2003). Hormones and competitive aggression in women. Aggressive Behavior, 29, 107-115. doi: 10.1002/ab.10041.

Coetzee, V., Re, D., Perrett, D. I., Tiddeman, B. P., \& Xiao, D. (2011). Judging the health and attractiveness of female faces: Is the most attractive level of facial adiposity also considered the healthiest? Body Image, 8 , 190-193. doi:10.1016/j.bodyim.2010.12.003.

Campbell, A. \& Cross, C. (2012). Women and aggression. In T. Shackelford \& V. Weekes-Shackelford (Eds.), The Oxford Handbook of Evolutionary Perspectives on Violence, Homicide, and War (pp. 197217). Oxford, NY:Oxford University Press.

Deaner, R. O., Goetz, S. M. M., Shattuck, K., \& Schnotala, T. (2012). Body weight, not facial width-to-height ratio, predicts aggression in pro hockey players. Journal of Research in Personality, 46, 235-238. doi:10. 1016/j.jp. 2012.01.005.

Fessler, D.M.T., Holbrook, C., Tiokhin, L.B., \& Snyder, J.K. (2014). Nonviolent physical risk-taking enhances the envisioned bodily formidability of women. Journal of Evolutionary Psychology, 12(2-4). doi: 10.1556/JEP-D-14-00009.

Gallup, A. C., \& Wilson, D. S. (2009). Body mass index (BMI) and peer aggression in adolescent females: an evolutionary perspective. Journal of Social, Evolutionary, and Cultural Psychology, 3(4), 356-371.

Geniole, S. N., Keyes, A. E., Mondloch, C. J., Carre, J. M., \& McCormick, C. M. (2012). Facing aggression: cues differ for female versus male faces. PLoS One, 7(1), 1-12.

Geniole, S. N., Denson, T. F., Dixson, B. J., Carre, J. M., \& McCormick, C. M. (2015). Evidence from metaanalyses of the facial width-to-height ratio as an evolved cue of threat. PLoS One. doi:10.1371/journal. pone. 0132726 .

Grant, V. J., \& France, J. T. (2001). Dominance and testosterone in women. Biological Psychology, 58, 41-47.

Haselhuhn, M. P., \& Wong, E. M. (2011). Bad to the bone: facial structure predicts unethical behaviour. Proceedings of the Royal Society of London B: Biological Sciences, 279(1728), 571-576. doi:10.1098/ rspb.2011.1193.

Haselhuhn, M. P., Ormiston, M. E., \& Wong, E. M. (2015). Men's facial width-to-height ratio predicts aggression: a meta-analysis. PLoS One, 10(4), e0122637. doi:10.1037/journal.pone.0122637.

Kramer, R. S. S. (2015). Facial width-to-height ratio in a large sample of commonwealth games athletes. Evolutionary Psychology, 13(1), 197-209.

Law Smith, M. J., Perrett, D. I., Jones, B. C., Cornwell, R. E., Moore, F. R., Feinberg, D. R., et al. (2006). Facial appearance is a cue to oestrogen levels in women. Proceedings of the Royal Society of London B: Biological Sciences, 273, 135-140. doi:10.1098/rspb.2005.3296.

Lefevre, C. E., Lewis, G. J., Bates, T. C., Dzhelyova, M., Coetzee, V., Deary, I. J., \& Perrett, D. I. (2012). No evidence for sexual dimorphism of facial width-to-height ratio in four large adult samples. Evolution and Human Behavior, 33, 623-627. doi:10.1016/j.evolhumbehav.2012.03.002.

Lefevre, C. E., Lewis, G. J., Perrett, D. I., \& Penke, L. (2013). Telling facial metrics: facial width is associated with testosterone levels in men. Evolution and Human Behavior, 34(4), 273-279. doi:10.1016/j. evolhumbehav.2013.03.005. 
Lefevre, C. E., Etchells, P. J., Howell, E. C., Clark, A. P., \& Penton-Voak, I. P. (2014). Facial width-to-height ratio predicts self-reported dominance and aggression in males and females, but a measure of masculinity does not. Biology Letters, 10, 1-4. doi:10.1098/rsbl.2014.0729.

Miyatake, N., Miyachi, M., Tabata, I., Sakano, N., Hirao, T., \& Numata, T. (2012). Relationship between muscle strength and anthropometric, body composition parameters in Japanese adolescents. Health, 4(1), 1-5. doi:10.4236/health/2012.41001.

Özener, B. (2012). Facial width-to-height ratio in a Turkish population is not sexually dimorphic and is unrelated to aggressive behavior. Evolution and Human Behavior, 33(3), 169-173. doi:10.1016/j. evolhumbehav.2011.08.001.

Pinhey, T. K. (2002). A research note on body mass, physical aggression, and the competitiveness of AsianPacific Islander adolescents in Guam. Social Biology, 49(1-2), 90-98.

Quist, M. C., Watkins, C. D., Smith, F. G., DeBruine, L. M., \& Jones, B. C. (2011). Facial masculinity is a cue to women's dominance. Personality and Individual Differences, 50(7), 1089-1093. doi:10.1016/j.paid. 2011.01.032.

Re, D. E., \& Perrett, D. I. (2014). The effects of facial adiposity on attractiveness and perceived leadership ability. Quarterly Journal of Experimental Psychology, 67(4), 676-686. doi:10.1080/17470218.2013. 825635.

Rule, N., \& Ambady, N. (2009). She's got the look: inferences from female chief executive officers' faces predict their success. Sex Roles, 61(9-10), 644-652. doi:10.1007/s1119-009-9658-9.

Sell, A., Cosmides, L., Tooby, J., Sznycer, D., von Rueden, C., \& Gurven, M. (2009). Human adaptations for the visual assessment of strength and fighting ability from the body and face. Proceedings of the Royal Society of London B: Biological Sciences, 276, 575-584. doi:10.1098/rspb.2008.1177.

Sowers, M. F., Beebe, J. L., McConnell, D., Randolph, J., \& Jannausch, M. (2001). Testosterone concentrations in women aged 25-50 years: associations with lifestyle, body composition, and ovarian status. American Journal of Epidemiology, 153(3), 256-264.

Stirrat, M., \& Perrett, D. I. (2010). Valid facial cues to cooperation and trust: male facial width and trustworthiness. Psychological Science, 21(3), 349-354. doi:10.1177/0956797610362647.

Stirrat, M., Stulp, G., \& Pollet, T. V. (2012). Male facial width is associated with death by contact violence: narrow-faced males are more likely to die from contact violence. Evolution and Human Behavior, 33(5), 551-556. doi:10.1016/j.evolhumbehav.2012.02.002.

Trebicky, V., Havlicek, J., Roberts, S. C., Little, A. C., \& Kleisner, K. (2013). Perceived aggressiveness predicts fighting performance in mixed-martial-arts fighters. Psychological Science, 24(9), 1664-1672. doi:10.1177/0956797613477117.

Trebicky, V., Fialova, J., Kleisner, K., Roberts, S. C., Little, A. C., \& Havlicek, J. (2015). Further evidence for links between facial width-to-height ratio and fighting success: Commentary on Zilioli et al. (2014). Aggressive Behavior, 41, 331-334. doi:10.1002/ab.21559.

Weston, E. M., Friday, A. E., \& Liò, P. (2007). Biometric evidence that sexual selection has shaped the Hominin face. PLoS ONE, 2(8), e710.

Zilioli, S., Sell, A. N., Stirrat, M., Jagore, J., Vickerman, W., \& Watson, N. V. (2014). Face of a fighter: bizygomatic width as a cue of formidability. Aggressive Behavior, 9999, 1-9. doi:10.1002/ab.21544. 